\title{
The American Phytopathological Society Elected and Appointed Officers, Representatives, and Committees for 2006
}

Council

President: J. H. Andrews

President-Elect: J. E. Leach

Vice President: R. D. Martyn

Immediate Past President: J. D. MacDonald

Secretary: D. T. Beadle

Treasurer: E. L. Stromberg

Treasurer-Elect: R. C. Rowe

Editor-in-Chief, Phytopathology: R. L. Gilbertson

Editor-in-Chief, Plant Disease: K. L. Bowen

Editor-in-Chief, Molecular Plant-Microbe Interactions: J. Stougaard

Editor-in-Chief, Phytopathology News: M. L. Daughtrey

Editor-in-Chief, APS Press: R. C. Gergerich

Senior Councilor-at-Large: A. H. Tally

Intermediate Councilor-at-Large: B. J. Christ

Junior Councilor-at-Large: W. F. Wilcox

Councilor of the Caribbean Division: J. K. Brown

Councilor of the North Central Division: R. Hammerschmidt

Councilor of the Northeastern Division: M. T. McGrath

Councilor of the Pacific Division: G. G. Grove

Councilor of the Potomac Division: T. A. Evans

Councilor of the Southern Division: G. J. Holmes

Ex officio: APS Foundation Rep.: A. R. Chase

Executive VP: S. C. Nelson

\section{Representatives}

American Association for the Advancement of Science: D. G. Gilchrist

American Institute of Biological Sciences: R. D. Martyn

American Type Culture Collection: C. T. Bull

Coalition on Funding Agricultural Research: J. L. Sherwood

Council for Agricultural Science \& Technology: T. B. Sutton

International Society for Aboriculture: D. Neely

International Society for Plant Pathology: Chair: R. S. Zeigler. G. S. Abawi,

R. A. Bennett, K. F. Cardwell, J. C. Correll, T. A. Evans, J. W. Moyer, R.

C. Ploetz, L. Sequeira, A. H. van Bruggen

International Union of Microbiological Societies: TBD

Mycological Society of America: C. M. Stiles

National Council for Science and the Environment: K. L. Pernezny

Society of Environmental Toxicology and Chemistry: M. Simini

Society of Nematologists: E. L. Davis

APS Historian: P. D. Peterson

\section{APS Foundation, Board of Directors}

Chair: A. R. Chase. Vice Chair: M. L. Gleason. Past Chair: D. E. Mathre. Secretary: W. E. Fry. Treasurer: E. L. Stromberg. J. Amador, C. J. D'Arcy, G. L. Schumann, Ex-Officio (non-voting): A. R. Records

Office of Electronic Communications (OEC) Board

Director: D. M. Eastburn. APSnet Feature Editor: M. L. Elliott. C. T. Bull, G. T. Church, G. G. Grove, P. M. Sforza, K. C. Steddom, Ex-Officio (nonvoting): D. Benson. Ex-Officio (non-voting): A. B. Baudoin

Office of International Programs (OIP) Advisory Board

Director: R. C. Ploetz. M. Babadoost, J. Fletcher, N. Grunwald, D. P. Maxwell, S. A. Miller, J. R. Steadman, Ex-Officio (non-voting): W. E. Fry

\section{Office of Industry Relations (OIR) Board}

Director: H. Morton. C. M. Becker, L. Fought, C. L. Kohls, G. P. Munkvold, B. D. Olson, B. Rao, C. T. Schiller, V. Spadafora, D. L. Thomas, ExOfficio Voting: M. R. Schwarz. Ex-Officio Voting: E. C. Tedford

Office of Public Affairs and Education (OPAE) Advisory Board

Director: D. J. Jardine. Communications Coordinator: J. P. Damicone. G. S. Abawi, C. R. Curtis, M. A. Draper, C. T. Schiller, Ex-Officio, APSnet Feature Editor: M. L. Elliott. Ex-Officio (non-voting): S. Coakley. ExOfficio (non-voting): J. D. MacDonald
Public Policy Board (PPB)

Chair: S. Coakley. Chair-Elect: J. Fletcher. J. H. Andrews, N. Carroll, S. Gold, J. E. Leach, J. D. MacDonald, R. D. Martyn, D. C. McGee, J. R. Steadman, R. E. Stuckey, Ex-Officio (non-voting): D. J. Jardine

Scientific Programs Board

Director: E. N. Rosskopf. Program Chair: J. E. Leach. Vice Chair: R. D. Martyn. Section Chairs: S. T. Adkins, M. J. Boehm, R. M. Bostock, C. T. Bull, A. K. Culbreath, J. E. Lundquist, Workshop Chair: D. G. Fernando. A. B. Baudoin, A. H. Tally

APS Publications Board

Chair: M. E. Daub. A. B. Baudoin, D. Benson, S. C. Bost, K. L. Bowen, M. L. Daughtrey, D. S. Egel, R. C. Gergerich, R. L. Gilbertson, J. Stougaard

Division Officers

Caribbean Division: President: J. Filgueira-Duarte. Vice President: $\mathrm{O}$. Moreno Valenzuela. Past President: C. M. Araya. Secretary-Treasurer: R. H. Brlansky

North Central Division: President: C. A. Bradley. Vice President: J. L. Beckerman. Secretary-Treasurer: L. J. Giesler

Northeastern Division: President: W. H. Elmer. Vice President: C. A. Smith. Immediate Past President: A. Gould. Secretary-Treasurer: D. R. Cooley. Councilor Elect: R. L. Wick

Pacific Division: President: B. J. Jacobsen. President-Elect: L. D. Porter. Past President: M. W. Olsen. Secretary-Treasurer: M. A. Yoshimura. Newsletter Editor: E. M. Bentley

Potomac Division: President: J. L. Norelli. Vice President: K. L. Everts. Immediate Past President: R. E. Davis. Secretary-Treasurer: D. P. Roberts

Southern Division: President: T. B. Brenneman. President-Elect: C. S. Rothrock. Vice President: C. A. Clark. Past President: D. Benson. Secretary-Treasurer: T. S. Isakeit

APS Press

Editor-in-Chief: R. C. Gergerich. Aquisitions Editor: T. A. Zitter. Senior Editors: A. M. Alvarez, C. T. Bull, B. J. Jacobsen, S. T. Koike, R. R. Martin, S. A. Miller, J. K. Pataky, T. C. Paulitz, C. P. Woloshuk, ExOfficio (non-voting): E. L. Stromberg

Illustrations of Plant Pathogens and Diseases: Chair: T.C. Paulitz. S. L. Giammaria, C. S. Rothrock, K. L. Schroeder

Standardization of Common Names of Plant Diseases: P. H. Flynn. B. A. Fortnum, C. A. Hollier, T. J. Michailides, N. J. Taylor

Editors of Molecular Plant Microbe Interactions

Editor-in-Chief: J. Stougaard. Senior Editors: G. Bloemberg, S. P. DineshKumar, P. Dodds, O. Geiger, G. Gheysen, F. Govers, M. Grant, B. MauchMani, R. P. Oliver, M. Parniske, H. B. Scholthof, M. Udvardi, L. L. Walling, F. F. White, Indexing Editor: T. Kommedahl. Associate Editors: J. Bakker, A. Becker, L. M. Ciuffetti, J. N. Culver, K. F. Dobinson, M. Gijzen, S. Gold, E. Journet, H. Kouchi, Y. Kubo, K. N. Lambert, G. J. Loake, I. M. Lopez-Lara, E. Martinez-Romero, Y. Moenne-Loccoz, M. Mudgett, U. Paszkowski, K. Pawlowski, J. M. Salmeron, F. Sanchez, K. G. Scholthof, K. Szczyglowski, N. J. Talbot, B. P. H. J. Thomma, I. Toth, J. Verchot-Lubicz, S. A. Whitham

\section{Editors of Phytopathology}

Editor-in-Chief: R. L. Gilbertson. Associate Editors: J. E. Adaskaveg, X. Chen, B. Ding, L. R. Gale, A. E. Glenn, R. C. Grube, J. S. Hartung, C. R. Howell, B. B. McSpadden Gardener, J. A. Rollins, W. Schneider, G. W. Sundin, W. W. Turechek, J. E. Yuen

Editors of Plant Disease

Editor-in-Chief: K. L. Bowen. Senior Editors: M. L. Gleason, F. A. Gray, A. P. Keinath, R. C. Larsen, M. T. McGrath, G. Newcombe, J. W. Pscheidt, J. C. Rupe, L. W. Timmer, J. Y. Uchida, S. N. Wegulo, Focus Editor: T. Kommedahl. Feature Editor: M. P. McMullen. Disease Notes Assigning Editor: R. E. Baird. Associate Editors: R. Creamer, A. Gould, H. M. Griffiths, G. E. Holcomb, W. Kirk, M. T. Mmbaga, H. R. Pappu, A. T. Ploeg, Z. A. Pretorius, K. W. Seebold, W. Uddin, R. J. Walcott, A. Westphal, P. J. Zambino, N. K. Zidack 
Editors of Plant Health Progress

Editor-in-Chief: D. Benson. Senior Editors: D. A. Cuppels, J. E. Funderburk, T. P. Kuhar, S. B. Martin, M. E. Matheron, G. W. Moorman, R. N. Raid, C. S. Rothrock, P. Timper, Ex-Officio, APSnet Feature Editor: M. L. Elliott. Ex-Officio (non-voting): R. C. Gergerich

\section{Editors of Plant Health Instructor / APSnet Education Center}

Editor-in-Chief: A. B. Baudoin. Senior Editors: E. J. Braun, A. Gould, N. Grunwald, M. A. Langham, S. J. Pethybridge, J. P. Stack, K. L. Stevenson, M. A. Sulzinski, E. C. Tedford, J. Xu

\section{Editors of Biological \& Cultural Tests}

Editor-in-Chief: S. C. Bost. Section Editors: C. R. Hollingsworth, C. S. Johnson, M. A. Newman, J. A. Wrather, T. M. Momol, R. P. Larkin, N. A. Tisserat, M. C. Black, M. E. Matheron

\section{Editors of Fungicide and Nematicide Tests}

Editor-in-Chief: D. Egel. Section Editors: P. Phipps, J. Miller, K. Everts, D. Langston, K. Seebold, P. Roberts, L. du Toit, B. Olson, A. Henn, C. Bradley, J. Rich, J. Buck, D. Cooley, R. Kemerait, B. Cline, A. Schilder, N. Lalancette, F. Wong, C. Becker

\section{Phytopathology News Advisory}

Editor-in-Chief: M. L. Daughtrey. A. Grybauskas, M. Lorito, K. O. Osikanlu, A. H. Tally

\section{Society Internal Relations Committees}

Awards and Honors: Chair: L. S. Thomashow. Immediate Past Chair: T. R. Gottwald. Vice Chair: C. E. Windels. M. E. Daub, M. B. Dickman, B. W. Falk, R. C. Seem

Committee on Committees: Chair: A. H. Tally. B. J. Christ, W. F. Wilcox

Financial Advisory: Chair: E. L. Stromberg. J. H. Andrews, T. A. Evans, R. C. Gergerich, J. E. Leach, R. D. Martyn, A. H. Tally, W. F. Wilcox

\section{Society General Policies Committees}

Biotechnology Impact Assessment: Chair: Y. Jia. Vice Chair: K. Ling. M. A. Bhatti, B. Guo, Z. Kanaan-Atallah, A. Pepper, S. D. Soby, Y. Wamishe, W. Wechter, Y. Yang

Collections and Germplasm: Chair: X. Chen. Immediate Past Chair: B. J. Goates. Vice Chair: C. M. Catranis. P. A. Berland, L. E. Cadle-Davidson, W. Chen, J. Chen, M. D. Coffey, F. M. Dugan, S. Li, D. L. Long, J. A. Thies, A. H. Yahyaoui

Early Career Professionals: Chair: J. K. O'Mara. Immediate Past Chair: S. J. Vasquez. Vice Chair: B. K. Scholz-Schroeder. M. E. Ambroson, M. C. Becktell, C. T. Bull, R. Bulluck, N. J. Catlin, A. E. Glenn, P. F. Harmon, Z. Kanaan-Atallah, L. M. Keith, P. R. Northover, K. Ong, M. E. Ordonez, D. G. Schmale, K. T. Schneider, V. Toussaint, M. R. Vazquez, N. R. Walker, C. M. Wistrom, Y. Zhao

Extension: Chair: L. J. Giesler. Immediate Past Chair: G. M. Leavitt. Vice Chair: D. K. Malvick. E. B. Adams, G. S. Bender, C. A. Bradley, T. W. Hall, P. F. Harmon, A. Henn, C. R. Hollingsworth, T. A. Jackson, Y. Luo, J. C. Mertely, C. J. Mickler, N. A. Mitkowski, K. Ong, J. M. Rees, A. E. Robertson, A. C. Schilder, J. P. Stack, K. C. Steddom, A. U. Tenuta, S. J. Vasquez

Graduate Student: Chair: A. R. Records. Immediate Past Chair: D. G. Schmale. Vice Chair: B. A. Edmunds. R. M. Andrie, T. A. Bowers, M. Diagne, M. Van Wyk, K. M. Webb

Industry: Chair: J. A. Frank. Immediate Past Chair: M. R. Schwarz. Vice Chair: B. D. Olson. J. V. Florentine, L. Fought, V. Jurin, Z. KanaanAtallah, M. C. Klapproth, P. J. Kuhn, R. T. Lartey, G. P. Munkvold, C. L. Palmer, L. A. Payan, J. L. Riggs, C. T. Schiller, E. C. Tedford, B. Windsor, H. L. Ypema

Joint Committee of Women in Plant Path \& Cultural Diversity: Chair: M. E. Ambroson. Immediate Past Chair: C. Yandoc. Vice Chair: R. HernandezMartinez. A. A. Bacetty, M. A. Boudreau, C. T. Bull, R. Bulluck, C. Cowger, A. Den Breeyen, J. E. Fajardo, D. G. Fernando, J. A. Gigot, M. L. Heppler, N. Hyder, N. I. Khan, R. A. Marvelli, A. E. McClean, A. L. Mila, R. Onaolapo Olatinwo, F. Runa, E. Seyran, P. Sudarshana, C. M. Vera Cruz, S. N. Wegulo

Placement: Chair: J. E. Creasap. Immediate Past Chair: A. DeMarsay. S. O. Aigbe, B. J. Bush, J. F. Davey, E. N. Rosskopf, L. Ines Santamaria, T. V. Taylor, H. E. Velez

Private Practice: Immediate Past Chair: C. L. Kohls

Regulatory Plant Pathology: Chair: G. L. Peterson. Immediate Past Chair: P. I. Lewis. Vice Chair: N. K. Osterbauer. Vice Chair Elect: T. D. Riley, T. N. Boratynski, R. Bulluck, K. F. Cardwell, T. R. Gottwald, C. F. Harden, S. S. Hurtt, K. L. Kosta, R. F. Lee, E. V. Podleckis, B. L. Randall-Schadel, S. Rizvi, C. M. Sandlin, K. L. Smith, X. Sun, M. G. Tiffany, A. T. Tschanz, D. M. Woods, H. Xu, R. E. Ykema
Teaching: Chair: M. B. Riley. Immediate Past Chair: G. W. Hudler. Vice Chair: D. M. Eastburn. L. P. Berkett, K. D. Cox, P. H. Flynn, S. K. Gremillion, C. Lapaire Harmon, C. M. Kenerley, M. Skaria, C. M. Stiles

\section{Subject Matter Committees}

Bacteriology: Chair: D. Y. Kobayashi. Immediate Past Chair: S. He. Vice Chair: A. O. Charkowski. J. R. Alfano, J. D. Barak, A. J. Bogdanove, M. L. Brodhagen, C. T. Bull, W. Chun, J. M. Clifford, B. K. Duffy, Z. Flores, J. H. Graham, R. Hernandez-Martinez, K. Jakob, J. B. Jones, L. M. Keith, J. A. Kers, R. Loria, F. J. Louws, S. Lu, G. Luo, M. L. Miller, K. L. Newman, J. L. Norelli, A. R. Records, R. M. Reedy, N. W. Schaad, C. D. Smart, G. W. Sundin, S. B. Von Bodman, F. F. White, Y. Zhao

Biochemistry, Physiology \& Molecular Biology: Chair: C. D. Smart. Immediate Past Chair: L. J. Vaillancourt. Vice Chair: T. K. Mitchell. J. J. Choi, Y. E. Choi, L. M. Ciuffetti, M. B. Dickman, A. M. Fakhoury, J. E. Flaherty, T. L. Graham, D. A. Halterman, S. L. Hartney, Y. Jia, M. V. Kolomiets, D. Kumar, J. M. McDowell, B. B. McSpadden Gardener, S. Restrepo, U. Shankar Sagaram, W. Shim, S. A. Sukno, M. R. Thon, S. R. Uppalapati, J. D. Walton, D. S. Warkentin, R. P. Wise, C. P. Woloshuk

Biological Control: Chair: A. A. Collins. Immediate Past Chair: R. P. Larkin. Vice Chair: S. Kiewnick. H. K. Abbas, P. A. Backman, J. Borneman, W. L. Bruckart, C. T. Bull, S. Chen, A. M. Demers, S. S. Gnanamanickam, B. J. Jacobsen, M. S. Krause, D. Lakshman, J. E. Loper, M. Lorito, B. B. McSpadden Gardener, R. L. Melnick, F. A. Mrema, J. M. Raaijmakers, D. C. Sands, D. A. Schisler, S. F. Shamoun, W. Wechter, D. M. Weller, C. Yandoc, G. Y. Yuen

Chemical Control: Chair: R. C. Kemerait. Vice Chair: C. J. Mickler. S. A. Alexander, W. Chen, A. Cochran, D. R. Cooley, J. P. Damicone, L. Fought, M. F. R. Khan, C. L. Kohls, H. Morton, B. D. Olson, L. A. Payan, K. M. Tubajika, B. Windsor, L. E. Zang

Crop Loss Assessment and Risk Evaluation: Chair: D. G. Schmale. Immediate Past Chair: C. A. Hollier. Vice Chair: F. W. Nutter. P. D. Esker, G. Hughes, M. M. Kennelly, S. J. Lambert, D. L. Long, J. S. Miller, O. Modesto Olanya, F. W. Nutter, S. J. Pethybridge, K. C. Steddom, J. M. Stein, W. W. Turechek, C. R. Wilson, C. Yandoc

Diagnostics: Chair: T. D. Blunt. Immediate Past Chair: J. L. Chaky. Vice Chair: J. L. O’Mara. Y. Abou Jawdah, F. M. Assis Filho, W. O. Bliss, L. G. Brown, A. Fabritius, R. C. Hamelin, M. Hansen, C. Lapaire Harmon, E. W. Honeycutt, J. C. Jacobi, M. Marie Kennelly, L. Levy, D. K. Malvick, S. K. Marquardt, R. T. McMillan, C. Nischwitz, F. M. Ochoa-Corona, A. J. Palmateer, G. E. Ruhl, P. J. Shiel, K. L. Snover-Clift, C. L. Sutula, M. R. Williamson, S. Wright, H. Xu, K. A. Zeller

Diseases of Ornamental Plants: Chair: W. E. Copes. Immediate Past Chair: S. N. Wegulo. Vice Chair: C. L. Palmer. J. M. Byrne, G. T. Church, M. L. Daughtrey, J. L. Hall, J. R. Hartman, A. Henn, S. N. Jeffers, N. I. Khan, A. D. Martinez-Espinoza, R. J. McGovern, M. T. Mmbaga, R. P. Mulrooney, D. J. Norman, M. J. Pearce, K. A. Plumley, B. Rao, B. B. Reddick, C. Y. Warfield, R. L. Wick

Environmental Quality \& Plant Health: Chair: M. Simini. Immediate Past Chair: C. R. Krause. Vice Chair: C. R. Krause. M. A. Bhatti, M. S. Krause

Epidemiology: Chair: R. D. Magarey. Immediate Past Chair: E. D. De Wolf. Vice Chair: P. D. Esker. C. Cowger, N. S. Dufault, K. A. Garrett, C. R. Hollingsworth, J. E. Molineros, U. N. Nanayakkara, M. Nita, F. W. Nutter, P. A. Paul, S. S. Savary, H. Scherm, P. M. Sforza, G. E. Shaner, K. M. Tubajika

Forest Pathology: Chair: P. Bonello. Immediate Past Chair: P. J. Zambino. Vice Chair: M. Kim. D. N. Appel, C. L. Ash, K. S. Camilli, G. A. Chastagner, M. Chen, D. J. Collins, L. Englander, S. J. Frankel, E. M. Hansen, J. A. Hoff, J. Juzwik, G. Laflamme, W. L. MacDonald, R. E. Marra, C. Mohammed, J. G. O’Brien, D. M. Rizzo, S. F. Shamoun, W. C. Shortle, P. C. Spaine, J. K. Stone, J. W. Zanzot

Genetics: Chair: N. Grunwald. Immediate Past Chair: R. L. Bowden. Vice Chair: K. A. Zeller. T. B. Adhikari, R. G. Bhat, K. Chung, T. L. Friesen, S. L. Giammaria, B. Guo, S. D. Harris, R. Hernandez-Martinez, H. S. Judelson, M. R. Kerns, C. Khang, S. J. Klosterman, Y. Lee, M. A. PastorCorrales, B. Turgeon, P. Veronese, J. Xu, H. Xue

Host Resistance: Chair: C. R. Grau. Immediate Past Chair: M. G. Redinbaugh. Vice Chair: B. Guo. T. B. Adhikari, R. G. Bhat, J. Bonman, H. Bouzar, M. C. Carrillo, M. L. Carson, F. Correa, R. C. Grube, C. B. Hill, J. Hoogland, R. M. Hunger, S. Kang, A. Mengistu, S. Xavier Mideros Mora, M. A. Pastor-Corrales, J. J. Polashock, J. B. Rasmussen, B. J. Steffenson, J. A. Thies, G. E. Vallad, P. Veronese, K. M. Webb, H. Xue, A. H. Yahyaoui

Integrated Plant Disease Management: Chair: L. J. Chapin. Immediate Past Chair: T. M. Momol. Vice Chair: W. McFadden-Smith. Y. Abou Jawdah, L. P. Berkett, K. L. Everts, R. P. Larkin, J. J. Marois, B. J. Messenger, T. M. Momol, K. L. Pernezny, A. C. Schilder, S. M. Schneider, S. F. Shamoun 
Mycology: Chair: M. Skaria. Immediate Past Chair: C. Lapaire Harmon. Vice Chair: T. E. Chase. L. E. Cadle-Davidson, C. M. Catranis, K. D. Cox, J. C. Dianese, D. M. Eastburn, D. M. Geiser, A. J. Gevens, S. B. Goodwin, G. W. Hudler, S. A. Jordan, S. Rizvi, C. M. Stiles, P. J. Zambino

Mycotoxicology: Chair: C. P. Woloshuk. Immediate Past Chair: R. L. Brown. Vice Chair: J. Palumbo. H. K. Abbas, C. W. Bacon, B. J. Bush, Z. Chen, P. J. Cotty, A. E. Desjardins, R. Dill-Macky, A. M. Fakhoury, D. L. Funnell, A. E. Glenn, B. Guo, S. Herrero, R. Jaime-Garcia, M. V. Kolomiets, M. A. Mansfield, T. J. Michailides, E. R. Palencia, G. A. Payne, J. F. Plasencia, R. H. Proctor, B. M. Pryor, A. Schaafsma, W. Shim, C. P. Woloshuk, I. E. Yates, N. C. Zitomer

Nematology: Chair: K. Wang. Immediate Past Chair: J. B. Kotcon. Vice Chair: B. S. Sipes. A. A. Bacetty, R. Bulluck, S. Chen, J. Desaeger, R. A. Dunn, C. S. Johnson, N. Kokalis-Burelle, R. Levin, S. L. Meyer, T. L. Niblack, J. L. Padgham, M. D. Petrillo, G. L. Tylka, J. K. Welch, A. Westphal, L. Xing

Pathogen Resistance: Chair: F. P. Wong. Immediate Past Chair: M. T. McGrath. Vice Chair: W. F. Wilcox. J. P. Damicone, R. P. Kaiser, W. D. Koeller, D. B. Langston, P. I. Lewis, H. Morton, G. Olaya, N. Peres, G. Schnabel, L. W. Timmer, C. Xiao

Phyllosphere Microbiology: Chair: M. T. Brandl. Immediate Past Chair: G. Y. Yuen. Vice Chair: V. Toussaint. R. G. Bhat, T. J. Burr, K. Rae Everett, J. L. Jacobs, K. Jakob, K. B. Johnson, W. F. Mahaffee, P. S. McManus, R. T. McMillan, B. B. McSpadden Gardener, P. Pusey, M. Saunders, V. O. Stockwell, G. W. Sundin

Plant Pathogen and Disease Detection: Chair: R. R. Martin. Immediate Past Chair: R. T. McMillan. Vice Chair: M. G. Tiffany. P. H. Berger, R. G. Bhat, T. D. Blunt, J. D. Dominiak, A. Fabritius, S. L. Giammaria, L. Levy, S. K. Marquardt, V. A. Mavrodieva, J. H. McBeath, H. R. Pappu, P. J. Shiel

Postharvest Pathology: Chair: J. E. Adaskaveg. Immediate Past Chair: J. L. McEvoy. Vice Chair: E. C. Tedford. J. A. Bartz, B. D. Bruton, A. Cochran,
W. S. Conway, H. C. Forster, G. J. Holmes, W. J. Janisiewicz, L. Kanetis, Y. Kim, Y. Luo, J. Mercier, T. J. Michailides, D. B. Prusky, P. L. Sholberg, J. L. Smilanick, D. Sugar, K. M. Tubajika, C. Xiao, J. Zhang

Seed Pathology: Chair: B. L. Randall-Schadel. Immediate Past Chair: L. M. Shepherd. Vice Chair: W. L. Wiebe. A. M. Alvarez, K. S. Arthur, W. O. Bliss, C. C. Block, D. O. Burrell, L. D. Butler, A. C. Castro, J. L. Daniels, M. A. Draper, L. J. du Toit, A. Fabritius, R. L. Gilbertson, S. A. Heuchelin, P. T. Himmel, K. L. Johnson, W. Kaneshiro, H. Koenraadt, C. J. Kurowski, G. L. Lamka, M. Lusso, D. C. McGee, G. Olaya, A. Tayfour, A. B. Thornton, E. Vivoda, R. J. Walcott, S. K. Walker

Soil Microbiology and Root Diseases: Chair: L. E. Hanson. Immediate Past Chair: B. Spakes Richter. Vice Chair: A. T. Dyer. E. Blume, G. T. Browne, R. Bulluck, A. O. Charkowski, K. L. Everts, E. J. Fichtner, N. Grunwald, J. Hao, D. M. Huber, M. Jimenez-Gasco, C. D. Lewis, F. J. Louws, O. V. Mavrodi, N. Ochiai, S. Sanogo, K. L. Schroeder, S. R. Uppalapati, D. M. Weller, A. Westphal, L. Xing

Tropical Plant Pathology: Chair: R. H. Brlansky. Immediate Past Chair: F. M. Ochoa-Corona. Vice Chair: M. P. Grisham. S. O. Aigbe, W. O. Bliss, F. E. Brooks, L. A. Calvert, F. Correa, A. Kilaru, S. M. C. Njoroge, B. L. Randall-Schadel, B. S. Sipes, R. A. Valverde, J. A. Ventura

Turfgrass Pathology: Chair: S. Thomas. Immediate Past Chair: P. F. Harmon. Vice Chair: J. E. Kaminski. M. L. Agnew, T. D. Blunt, M. J. Boehm, L. L. Burpee, P. H. Dernoeden, D. Y. Han, G. Jung, M. Kennelly, U. N. Nanayakkara, E. B. Nelson, S. R. Parker, D. H. Perry, B. Rao, S. Thomas, M. Tomaso-Peterson, L. P. Tredway, H. C. Wetzel

Virology: Chair: A. V. Karasev. Immediate Past Chair: S. T. Adkins. Vice Chair: R. W. Hammond. J. A. Abad, F. M. Assis Filho, J. D. Bradshaw, M. E. L. Burrows, A. B. Cole, R. C. French, S. F. Hanson, A. M. Harness, S. Hogenhout, A. M. Idris, M. A. Langham, R. T. Lartey, D. J. Lewandowski, K. Ling, U. K. Melcher, L. G. Nemchinov, H. R. Pappu, N. A. Rayapati, N. L. Robertson, D. Robertson, G. Roy, W. Schneider, R. A. Welliver 